\title{
Parametric Patchwork
}

\section{Advancing the Development of an Organic Photovoltaic Carrier System through Various Computational Methods}

\author{
Timo Carl ${ }^{1}$, Markus Schein ${ }^{2}$ \\ ${ }^{1}$ University of Kassel ${ }^{2}$ School of Art, Kassel \\ ${ }^{1}$ tcarl@asl.uni-kassel.de²markus-schein@uni-kassel.de
}

This paper presents a strategy for implementing computational methods in education to solve specific project related research challenges. In our case, we investigate novel solutions for Organic Photovoltaic Carrier Systems. Therefore, environmental forces (especially the sun and shade) are important design drivers in all projects. Whilst the individual projects are limited to one semester, it is our aim to advance and accumulate these patches within a longer-term research strategy. Especially design-build projects that include digital fabrication often require a skillset not always available in a design studio environment. Providing simple parametric patches frees up time for creative investigations and allows tackling projects that are more complex. In the following, we will present and discuss a series of patches developed over the course of five projects that became our case studies. We conclude, by identifying relevant aspects that might be generalized and evaluate our insights for others.

Keywords: Computational Design, Parametric Design Strategies, Environmental Design Parameters, CAAD education

\begin{abstract}
INTRODUCTION AND RESEARCH CONTEXT
The shape of a building can be understood as the outcome of formative processes and comes about through the interaction of internal rules and external (morphogenetic) pressures (Kwinter 2003). Utilizing environmental parameters like sun and wind to inform and argue design decisions are a wellestablished approach within architectural history. Moreover, incorporating environmental forces as design drivers into the design process results in a more holistic design, challenging the separation of design and energy into opposite camps (Griffin and Millet
\end{abstract}

1984). The sun, in particular the phenomena of light and shadow are important variables, with the potential to connect buildings with their surroundings or to inform the façade design of build structures. Lastly, exist a variety of design methods - computational and others - that show a high potential to incorporate environmental and thus solar aspects in the design process.

Our specific research interest within this broader field is the integration of organic photovoltaic (OPV) cells as a means to combine energy production with passive shading and cooling. More important, our 
aim is to create a lightweight and highly adaptive carrier system that addresses both ecological and aesthetic concerns, because we believe that sustainable energy production within the build environment needs to be functional and beautiful at the same time. Consequently, such an approach requires both quantitative and qualitative design methods to reveal its inherent potentials.

In the following, we will outline five different ways to aid designing novel OPV carrier structures, based on 3D thermoformed modules (thin glass or polycarbonate sheets). All five case studies emerged over the last three years. The performance of these systems is influenced through environmental forces (sun, wind, rain) and has been developed through a patchwork of different computational and analogue design methods. Each patch addresses a specific, but as well overlapping aspect of our overall research interest, as the projects that gave birth to these methods, differed in scope, scale and available timeframes.

Academic Context. The common modus operandi for the presented research projects were design-build and design research teaching formats. Hence, ensue different timeframes and chronological sequences in comparison to traditional, noneducational research projects, as extensive research tasks have to be distributed among different teams and over a longer academic period. This is especially demanding within interdisciplinary teams. For instance, architects, designers and engineers have very different time budgets available for design built projects, ranging from a few hours until a couple of days per week. The patch concept that we are introducing allows us to distribute tasks with varying levels of difficulty and to respond to these challenges with a wide range of design methods.

Thus, we can develop longer-term and more complex projects with students who are only available for shorter periods, who have different capabilities and may be still in the process of differentiating their interests and preferred fields of investigation. Especially, when it comes to associative geometry modelling strategies, usually not all students come and want to be equipped with an adequate skillset. Thus, it becomes necessary to work with smaller algorithmic patches, supporting and amending analogue methods, instead of developing a single consistent global modelling strategy.

Modelling Strategies. A common ground for our design research teaching methods is the constant interweaving and interlinking of the physical and the virtual. Concerning digital modelling, we employ all three approaches as defined by Mark Burry (2003): Explicit geometry modelling, parametric modelling and associative geometry modelling. Especially the last category that informs and generates elaborate geometry by means of environmental forces is essential to this kind of research and seems hard to be substituted by analogue means.

However, it is often helpful to fall back on the rich tradition of physical form-finding strategies based on material behavior and physical laws - also known as

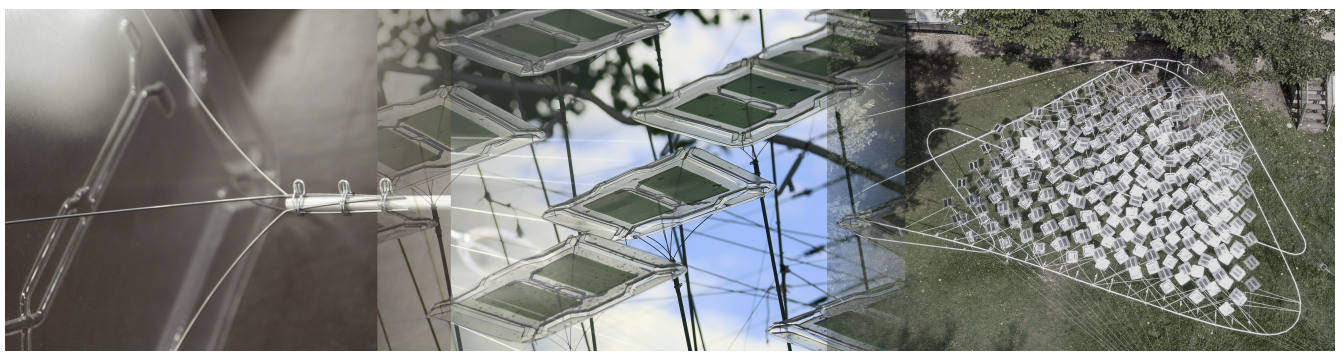

Figure 1

Cloud-like OPV carrier system in case study 1 
Figure 2

Impilcit tactile

knowledge is

transformed into

explicit insights by

geometrical

analysis and

constraint

formulation
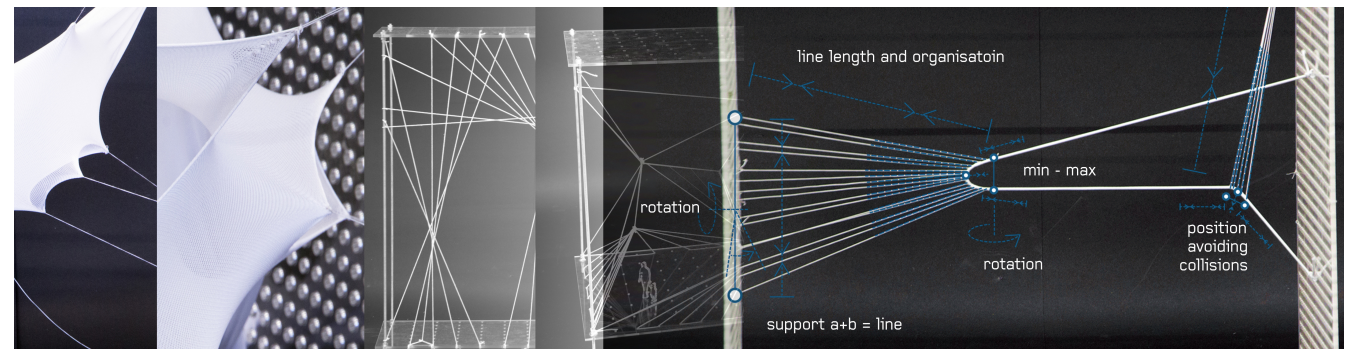

material computing (de Landa 2010) - as point of departure. The capacity to 'think with the hands' as understood by Otl Aicher (Aicher 1991) in combination with explicit modeling strategies is often appropriate enough to unlock and pursue novel solution spaces. The results of such methods may lack a certain precision in quantitative evaluation. However, they support design creativity and often yield rewarding returns in finding novel and unexpected solutions for a given problem (e.g. designing a carrier system with the gestalt of a solar cloud, see figure 1).

Knowledge Modelling. In what follows, we will introduce the concept of parametric patches by presenting five loosely connected educational designresearch and design-build projects around the topic of light-weight carrier systems for organic photovoltaic cells, for their integration into architectural structures.

The firs key issue concerns the implementation, respectively the development of adequate methodical patches and their integration within a single project. Subsequently, the second key issues is to identify and to pass on relevant knowledge across varying projects and to different participants.

Considering this, we will identify and compare the most successful benefits of the different analogue and digital patches, discuss their interlinkage and our knowledge modelling strategies. Finally, we will try to postulate for a more general but loose methodical framework for our future design-research studios, while arguing against a specific approach. This would oppose a universal parameter common to all design problems, namely their fundamental uniqueness (Rittel, Webber 1972).

\section{PARAMETRIC PATCHES CONCEPT}

We termed the combination of loosely connected design methods parametric patchwork. Each the design activity can be understood as parametric design, being analogue or digital, physical or virtual. Thus, we are preparing a common theoretical and methodical ground helping to gain and organize explicit knowledge from, as well implicit strategies, as depicted in figure 2. Noteworthy is the emergence of the parametric patch concept itself, as it was not a top down approach, but rather gradually developed from the analysis of various prior 'parametric' design projects and the derivation of successful methodical elements - meta-patches, so to speak.

We will exemplify our concept introducing five of these parametric patches more detailed. All case studies investigate the development of a carrier system for OPV-cells, trying to respond to the unique properties of those cells: being aesthetically and physically light, flexible, translucent, and customizable in different shapes and colors and with a low primary energy demand.

\section{DESIGN RESEARCH GOALS AND DRIVERS.}

The ecologically and socially urging demand of a permeatingly integrating the production of sustainable energy into our built environment, already has had big impacts - and not only positive ones. Our primary motivation is to demonstrate, that sustainability and aesthetics can go hand in hand and that multi- 


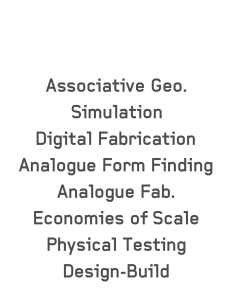

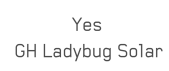$$
\text { Yes }
$$$$
\text { GH Ladybug Solar }
$$

Yes

$$
\begin{gathered}
\text { Case Study } 2 \\
\text { Yes } \\
\text { GH Ladybug Solar } \\
\text { asercutting, SLA Pr } \\
\text { Local } \\
\text { Vacuumforming } \\
\text { customized } \\
\text { Yes } \\
\text { Yes }
\end{gathered}
$$

functional approaches may lead to alternative design paths.

Currently, we focus on the design of a lightweight structure for OPV cells integration, comprised of three-dimensionally deforming sheets, suitable for different materials (glass, plastics, etc.). This approach includes geometric variation for both the individual carrier module, but also for the overall aggregate system of modules. Those can be produced in series, but also highly individualized in relation to the simulation of physical parameters. These design drivers can include solar radiation, shading patterns, wind or rain flows.

Combined with the unique properties of OPVcells, this approach spans a field of applications (figure 3) ranging from small-scale and mid-sized street furniture and urban interventions, façade systems towards larger architectural structures.

\section{PATCH A - SERIAL PARTS EMBEDDED IN A CUSTOMIZED PARAMETRIC MODEL}

Patch $A$ is exemplified through case study one named 'Solar Spline', an interdisciplinary design build and research project, that was carried out in four month time by the authors. It is a parametric patch, but essentially non-customizable. This strategy deliberately develops serial parts for a non-serial, adaptable structure for to reduce the qualitative complexity of a holistic design and manufacturing process (figure 4).

The serial element is a thermo-formed sheet from $0.5 \mathrm{~mm}$ polyethylene foil, serving as a temporary ultra-lightweight carrier for OPV-cells in a new type of sun sail, the Solar Spline (Carl, Schein 2018). The basic design features of this element consists of polygonised, undulating folds that stabilize the carrier sheet itself which function is to embed the OPVcells and all necessary electrical cables. Three hundred of these serially produced elements incorporate within the primary lightweight tensile structure of the Solar Spline. On a detail level, thin steel wires con-
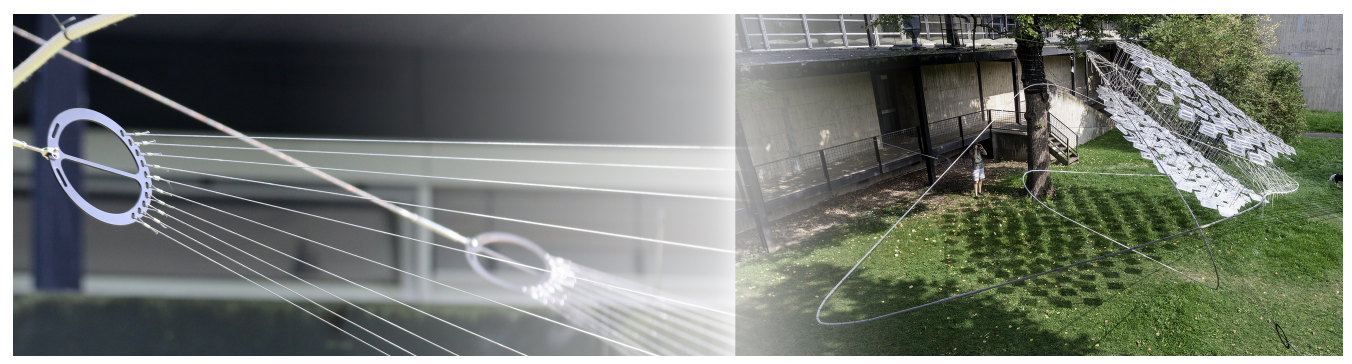

Figure 4

Arrays of serial parts within a flexible, adaptable and customizable tensile structure 
Figure 5

Sheet detail in final structure with printed joint(s), Digital modell of joints, Printed joints

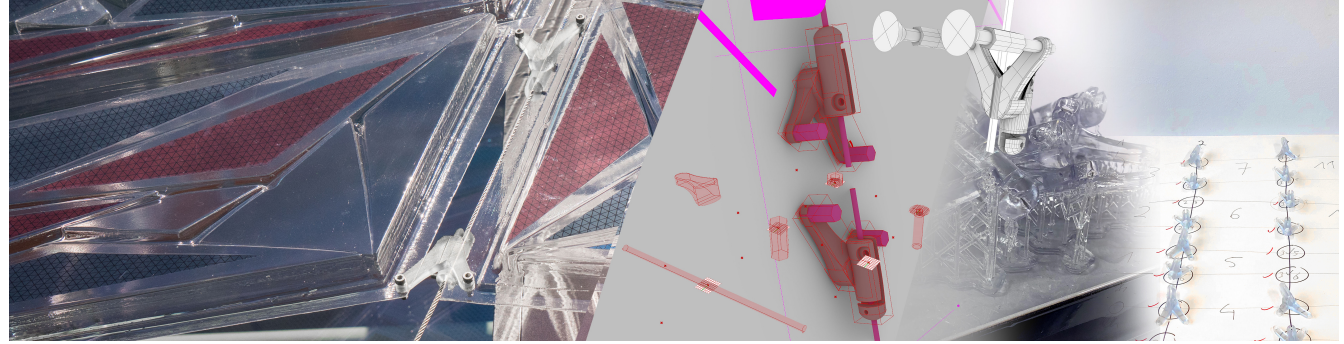

nect the serial OPV carrier elements to a secondary structure. The primary structure was represented in the parametric design model and informed by explicitly modelling (varying anchor amounts and positions) in combination with dynamic relaxation and solar radiation analysis. This way, the tensile structure itself could undergo a series of global transformations, whereas the carriers simply changed in amount and orientation, thus requiring no further adaption of shape, joints, etc.

Apart from the major advantage described above, this patch provided valuable proof for the potentials of combining serial and individualized elements in one workflow. Nevertheless, the project posed also related challenges, concerning the (virtually) linking of absolute and relative parameter and the question of how to construct accordingly in the physical realm.

\section{PATCH B - MATERIAL EXPERIMENTS, LOW- COMPLEXITY MODELLING AND DIGITAL FABRICATION}

The basic idea of patch $B$ is to express the results of simple physical experimentation into material behavior through simple digital modelling strategies and subsequently using basic digital fabrication techniques for production. Thus, providing a workflow of comparatively low instrumental complexity and therefore high accessibility. For exemplification, we have chosen an intense two-week design research and build seminar dealing with digital workflows.
Physical model experiments on material behavior of polycarbonate $(\mathrm{PC})$ were the point of departure for this patch. The aim was to develop and to evaluate various 3D-patterns that stabilize thin sheets of PC. The principles of the most successful tests were transferred into a parametric setup. This allowed for customized and serial parts simultaneously. Therefore, patch $B$ is a logical extension of the previously serial carrier elements into an individual facade system.

The digital modelling tools provided and employed within a Grasshopper / Rhino environment combined a surface based design approach (Killian 2006). A base surface is used to determine global geometrical parameters through a morph box strategy. The morph boxes generate variations of the basic shapes applicable on different surface geometries. As demonstrator, a four square meter large demonstrator was fabricated by rather simple rapid prototyping techniques. The Stereolitographie Apparatus (SLA) technology was used to manufacture the joints. A custom configured section component generated laser-cut geometry to build the molds for thermoforming the PC-sheets.

The design result was the prototype of a larger carrier system, with an architectural material in a real world setting, an enhancement of our first carrier system. However, during the process, we also experienced the typical disadvantages of the morph box tool, such as poor control of geometry in detail and the adjustment of standardized parts with customized elements. In contrast to the first case study, this was not addressed in depth by developing ad- 
equate construction details. In addition, one general flaw of the concept concerned the very loose connection of material experiments with the digital modelling strategy. An intermediary step (e.g. roughly estimating material stresses and expressing them by minimum section measures, angle constraints etc.) could override the morph box outputs, and would have improved this approach considerably.

\section{PATCH C - SOLAR ANALYSIS RESULTS AS DESIGN DRIVER}

We consider this patch as a digital tool and it consist of two elements. It is developed for easy integration of solar analysis and shading simulation data into design processes. Simultaneously, it demonstrates how these data can be straightforwardly inform geometry within a digital parametric setup. This solar analysis driven patch originates from an applied design research seminar about associative geometry modelling.

The tool implements as a grasshopper description, using built-in grasshopper components and Mostapha Sadeghipour Roudsari's Ladybug add-on. On the input side, weather-data and surfaces represent general or more detailed architectural geometries in relation to distinct environmental conditions. At the output side, common radiation analysis results are represented numerically and graphically. A short custom extension is added to calculate solar gains according to photovoltaic cells efficiency.

The sun vectors - one of the analysis parameters are used to demonstrate how a general setup for such an approach can be executed. Therefore, the input surface is subdivided into polygonal grid and further detailed into simple geometrical modules that populate the surface. The degree of opening of these modules is dependent on their orientation to the sun vector. The more they indicate to the sun, the closer the opening becomes, or vice versa.

As such, a behavior can easily be interpreted as south or north façade system. Moreover, it exemplifies our underlying didactic motivations. Applying this patch to their individual project work, students experience hands on that environmental forces can become a meaningful design driver. Either indirect, as tool to understand and simulate the environmental impacts of a project or directly by integrating it into an associative geometry model.

\section{PATCH D - INTERPRETING ENVIRONMEN- TAL FORCES THROUGH GEOMETRIC CON- STRAINS}

This patch compares to the first one (patch A) and is more of a design mindset or strategy, than a distinct tool to use. It aims is to avoid physical simulation strategies and substitute them through geometric constraining. Simultaneously, interfaces for later integration of such strategies are carefully planned. The underlying idea is to shift resources from the time consuming setup of physical parameter modelling. Instead, we make them available for design conception in the earlier stages of (digital) design processes. We applied this patch for the first time as a clearly perceptible strategy during a design study within a joint research project for the roofing of a bus-shelter with integrated solar infrastructure.

The goal of this study is to solve performative and practical design challenges within a single associative design framework. This includes solar shading, wind and rain protection, drainage and the detailed integration of large thermo-formed modules into a supporting tension-cable net structure. All geometry in the current model relates to a simplified representation of this cable net (figure 06 ). For the moment, geometric constraints generate the net that later on will link to simulation parameters, considering material properties and physical laws. Drainage of water is accomplished through a shingle topology that extends in two directions. The edges of the carrier sheets fold up and down to form a more rigid wind and watertight roof structure. Furthermore, their parametric setup is open to include other parameters such as roof angle or inclination towards the sun.

The concept of this patch is an integrated one. On one end, it allows to represent and develop a de- 
Figure 6

Simple analysis (of a global shape),

synthesis

(associative

geometry

modelling) and

evaluation (of the

refined surface)

sequence by the

provided

grasshopper

ladybug definition

Figure 7

Associate elements

include columns,

cable-net,

shingle-like

polycarbonate OPV

carrier sheets.

Modelled in the

Rhino/Grasshopper

environment and

ready to interface

with more

elaborate

modelling

strategies that are

based on Kangaroo,

Karamba, Ladybug

and potential

CFD-Simulation
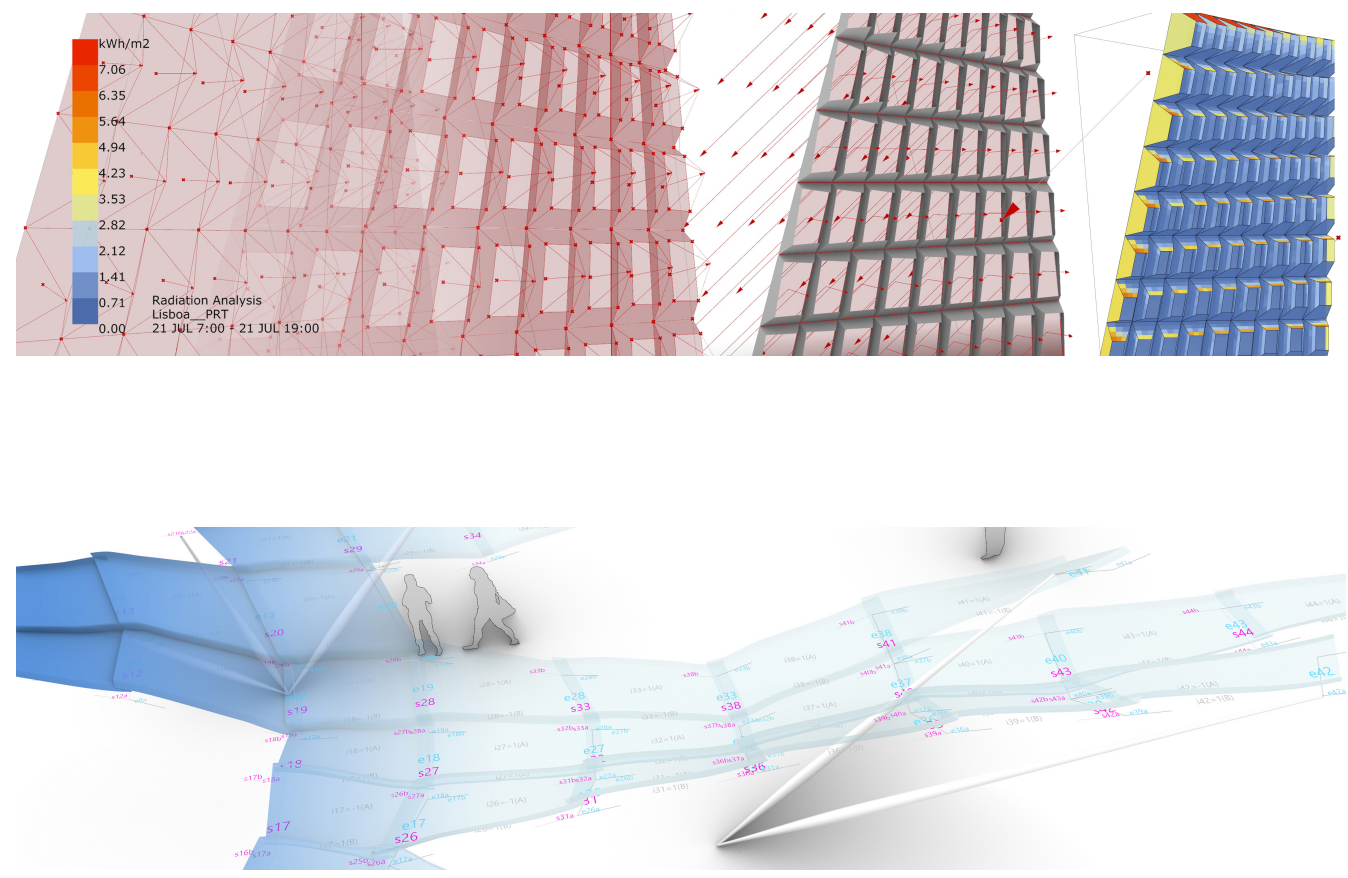

sign idea. On the other end, it is also open enough to accommodate other patches that have been developed previously gradually. However, the later aspect still needs to be evaluated, as it is a work in progress.

\section{PATCH E - LOOSE CONNECTIONS BETWEEN DIFFERENT PERFORMANCE PARAMETER MODELS}

The last patch, presented in this paper, responds to the fact that interfacing different digital modelling strategies and binding them together in one seamless digital design process may severely restrict creative exploration and prominently influence design decision making by forcing it early into a linear causal process chain.

To avoid this, the concept of patch E deliberately renounces to connect different performance mod- elling approaches or even to bring them into the same modeling environment. Instead, they are considered as memes, spreading from the virtual-digital realm to the virtual-analogue one and vice versa. This concept emerged from our most recent design research projects dealing with the design of urban solar structures.

One student team within this project was enthusiastic enough about performance driven digital design to find a generative approach representing their design idea of a circular array of wing-like OPVcarrier modules (figure 07). Besides explicit geometry modelling, parametric descriptions and some blender-based physical simulations, their design process started with two- and three-dimensional vector fields generating spatial organization schemes for the OPV carrier elements. Both the overall configuration, as well as the detailed morphology of the in- 


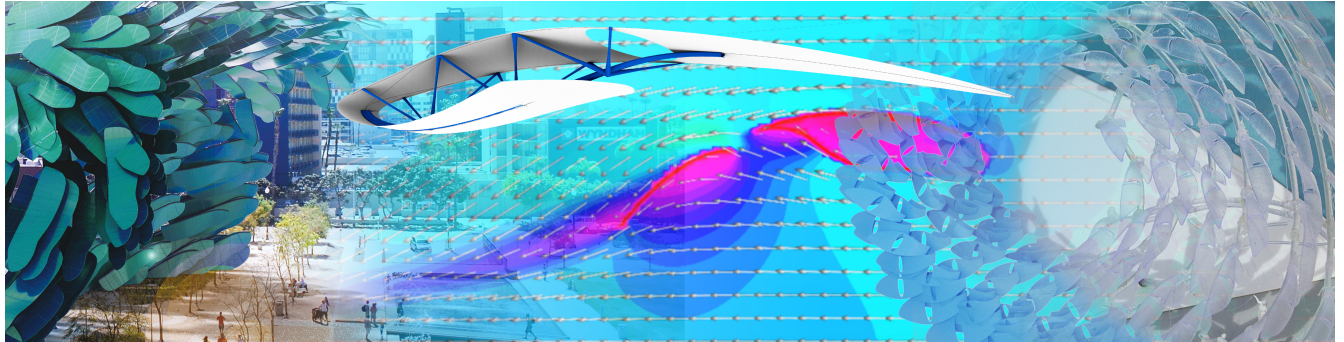

dividual element are optimized to funnel cool air towards the ground with computational fluid dynamics (CFD) software. This approach combines cooling through wind flows with cooling through passive shading. Solar radiation analysis informed the orientation of the carrier modules. Finally, the team used Topopt (Bialkowsky 2018) and Kangaroo (Piker 2015), two Grasshopper add-ons for topological optimization and dynamic relaxation, to refine the shape of joints and other elements of the primary structure. There was a clear benefit for the design team using this patch. However, if during the design process too much emphasis on interfacing different modeling approaches or interpreting their results without abstraction the design process would run the risk of being stuck by trying to formally connect them. The

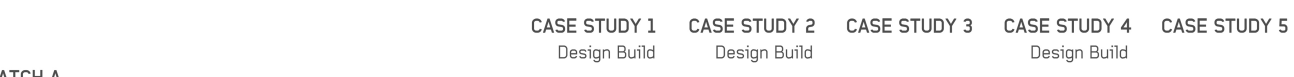

Serial Elements represented in Parametric Model

PATCH B

Simple Material Experiments

Low-Complexity Modelling

Low Complexity Digital Fabrication

PATCH C

Solar Analysis $=$ Design Driver

PATCH D

Envir. Forces inform Geometric Constraints

PATCH E

Expanded Investigations into Performance Driven Design concept of understanding different performance parameters also in a fuzzy, creative way turned out to be the most prolific design driver in this project.

\section{CONCLUSIONS}

Before drawing any conclusion, we caution that a comparative analysis of the presented methods, applied during five diverse design-research projects that become our case studies, is probably not enough to generalize our findings into ready rules. The number of patches chosen is limited and diverse, addressing different levels of integration and therefore evaluation. With hindsight, patch $B$ and $D$, including various different patches, might have been better subdivided or revised as design strategy

Figure 8

Solar energy

harvesting

combined with

shading and wind

driven cooling as

large urban

sculpture. Student

Team: Fabian Braun, Annemarie Sprunck and Michael

Schreiber

Figure 9

Comparison of patches and their respective implementation into the four design-research projects 
for this paper. Therefore, we will evaluate a work in progress, share our insight and identify the most valuable take-outs.

A major benefit of our patchwork approach is the capacity to develop a specific aspect paramount for the success of a design-research studio or seminar in a relatively short timeframe. Once a toolset is established and implemented successfully into a common modeling environment, there is the possibility of implicitly interfacing with other patches of follow up project work. Table XX provides an overview of the discussed patches and their respective degree of implementation in our five case study projects, ranging from marginal to medium and strong.

Frist, a major observation concerns the interfacing of these different patches. For this to work, each patch must have a clearly defined scope, as well as start and endpoint. Otherwise, it would require the simultaneous development of its predecessor and/or successors patches at once (e.g. for a seamless digital workflow, linking design fabrication data in a single associative model).

Instead, we advocate providing the freedom to experiment, modify and develop discrete patches addressing a single specific problem at hand. In our experience, it might be even favorable for design creativity to use only a select amount of computational methods, as this leaves more space for explorations outside the usual toolbox. The logical consequence of reducing the time spent for modelling design problems in all their complexity is that more space is available to reflect and design the overall research process to produce a more targeted output.

Second, accessibility is a key issue. To keep hurdles low and the application of parametric tools intuitive for students. Conceptualize a patch as a clearly identifiable strategy and provide the according toolset, respectively explanations and examples. Therefore, the interfaces between single patches should not be considered in a flow chart or programming logic. This observation refers to one of the key issues, we mentioned at the beginning in the paragraph knowledge modelling. Instead, we rec- ommend connecting different patches more loosely to circumvent various technical hurdles to free up resources to pursue relevant design opportunities. This will most probably involve mixing digital with analogue methods.

From a teaching perspective, the most demanding projects are those that try to implement a seamless digital design chain. If a research processes develops beyond the prototypical stage, the requirements towards a design model (complexity, its quantitative reliability, the scope of parameter to be represented) become more extensive and rigorous, redirecting a lot of resources back to the modelling process itself.

So, we say yes to a seamless digital design chain in an educational project only if we find a clear and self-evident way to organize a design process methodically from the beginning to the end. Moreover, we recommend also keeping an exit strategy to leave a sequence of logically combined patches, if it does not produce the required results.

Third, it is most beneficial if participants of previous projects and seminars continue in the consecutive semester. They can pass on instrumental and methodological knowledge about the patches already developed. In addition, they can provide valuable insight about the benefits and shortcomings of these patches and their usefulness for the current design investigations. In short, planting older seeds of knowledge (as information does not equal knowledge) into the current project is crucial. If no form previous semesters push on, this is accomplished in form of scientific conferences, lectures and presentations made available to the students.

Finally, we conclude that the digital patchwork approach presented previously turned out to be a good fit for the design research projects presented in this paper. With hindsight, we can say that combining, adjusting and modifying different patches of digital modelling methods according to different objectives was rather easy to implement. We hope that future work will deliver additional insight into the capacity of these patches to negotiate the specifics of 
a single design task while maintaining their generic nature of interfacing with other patches.

\section{AKNOWLEDGMENTS}

The design-build projects were made possible through the generous support of Opvius $\mathrm{GmbH}$, Carl Stahl GmbH and the 'Lehre für eine Nachhaltige Universität' Funding. We would like to thank Max Eschenbach for his support as teaching assistant in case study 3. We thank especially all our students who participated in the case study projects for their hard work and inspiration: Fabian Braun, Wassim Daaboul, Mahmoud Dames, Harun Faizi, Grisha Göbel, Grischa Göbel, Elena Mateev, Steffen Och, Mario Scherf, Annemarie Sprunck, Lisa Schreiber, Michael Schreiber, Ahmed Teftafeh,Tuantai Truong,

Bastian Wiesel. Department of Experimental Design and Construction (eek) - University of Kassel - www.uni-kassel.de/go/eek Digtal Fabrication Lab (DDD) - School for Art Kassel www.kunsthochschulekassel.de/personen/personendetails/person/schein-markus.html

\section{REFERENCES}

Aicher, O 1991, analog und digital, Ernst und Sohn, Berlin Burry, M 2003, 'Between Intuition and Process: Parametric Design and Rapid Prototyping', in Kolarevic, B (eds) 2003, Architecture in the Digital Age - Design and Manufacturing, Spon-Press, New York, p. $149 f$.

Carl, T, Schein, M and Stepper, F 2018 'Solar Spline Expanding on Traditional Sun Sail Typologies and Frei Otto's Lightweight Approach with the Help of Computational Design Procedures', Proceedings of the eCAADe 2018, Lodz

Griffin, F and Millet, M 1984, 'Shady Esthetics', Journal of Architectural Education, 37(3/4), p. 43

Kilian, A 2006, Design Exploration through Bidirectional Modeling of Constraints, MIT , Cambridge

Kwinter, S 2003, 'Who', in Kubo, M and Ferré, A (eds) 2003, Phylogenesis: FOA, Actar, Barcelona, pp. 96-99

Rittel, H 1972, 'Planen Entwerfen Design. Ausgewählte Schriften zu Theorie und Methodik', in Reuter, W (eds) 1972, Dilemmas in einer einen Theorie der Planung, Kohlhammer, Stuttgart, p. 22

[1] www.cluster.eu/2010/10/08/materialism-for-archite

cts-a-conversation-with-manuel-delanda/ 